\title{
Fusion of CTA and CTB gene to immunogen significantly enhances the immunogenicity of DNA vaccine
}

\author{
$X \operatorname{Ren}^{1 *}, Y W^{1}{ }^{1}, X X i e^{2}, J X u^{1}$ \\ From AIDS Vaccine 2012 \\ Boston, MA, USA. 9-12 September 2012
}

\section{Background}

Cholera toxin and its two subunits (CTA,CTB) have been intensively investigated as mucosal adjuvants for protein based vaccine. In this study we evaluated the adjuvanticity of CTA and CTB in modality of either mixing their encoding plasmids with DNA vaccine or fusing their encoding genes to immunogen encoding gene.

\section{Methods}

DNA and recombinant vaccinia vaccines expressing HIV-1 $\mathrm{AE}$ strain tat, rev, intergrase(C-half), vif, nef fusion gene (designated as TRIVN) have been constructed. For the construction of fusion gene of CTA/CTB to TRIVN, overlapping PCR was employed to link CTA/CTB and TRIVN gene, the fused genes(TRIVN-CTA and TRIVN-CTB)were cloned into eukaryotic expression plasmid vector(pSV1.0). Six groups of female BALB/c mice were immunized with mock control, pSV-TRIVN, pSV-TRIVN-CTA, pSVTRIVN-CTB, pSV-TRIVN mixed with CTA or with CTB respectively in a DNA priming-recombinant vaccinia boosting regimen. Two weeks after the final injection, mice splenocytes were collected and IFN- $\gamma$ ELISPOT assay were used as readout for specific T cell response. Statistical analysis was performed by using Prism5.0 software.

\section{Results}

Our data showed that all constructed plasmids are capable of efficiently expressing their inserted genes. All groups immunized with vaccines raised significant more $\mathrm{T}$-cell response than mock control. T-cell responses elicited by pSV-TRIVN-CTB(1548 \pm 330 SFCs/106splenocytes) and
pSV-TRIVN-CTA (1642 \pm 514 SFCs/106splenocytes) were significantly higher than that by pSV1.0-TRIVN (520 \pm 150 SFCs/106splenocytes), pSV1.0-TRIVN mixed with CTA (692 \pm 220 SFCs/106splenocytes) and pSV1.0TRIVN mixed with CTB (734 \pm 240 SFCs/106splenocytes). Though TRIVN-CTA and TRIVN-CTB fusion vaccines mounted comparable level of total IFN- $\gamma+\mathrm{T}$-cell responses, only TRIVN-CTB elicits significantly T-cell responses against Tat, which is a subdominant component in the fusion immunogen. No significant differences were observed among groups inoculated with TRIVN alone or adjuvanted by CTA/CTB subunit proteins.

\section{Conclusion}

CTA and CTB could serve as potent adjuvants for DNA vaccine in immunogen-CTA/CTB fusion modality. Compared with CTA, CTB may enhance T-cell responses against subdominant epitopes in the immunogen and broaden the $\mathrm{T}$-cell immune responses.

\section{Author details \\ ${ }^{1}$ Fudan University, Shanghai, China. ${ }^{2}$ SuZhou University, SuZhou, China.}

Published: 13 September 2012

doi:10.1186/1742-4690-9-S2-P8

Cite this article as: Ren et al:: Fusion of CTA and CTB gene to

immunogen significantly enhances the immunogenicity of DNA

vaccine. Retrovirology 2012 9(Suppl 2):P8.

${ }^{1}$ Fudan University, Shanghai, China

Full list of author information is available at the end of the article

(c) 2012 Ren et al; licensee BioMed Central Ltd. This is an Open Access article distributed under the terms of the Creative Commons 